\title{
Synthesis and Aggregation Properties of Salts Based on Aminomethylated Calix[4]resorcinols and 1-Aminoethylidenediphosphonic Acid
}

\author{
Liliya I. Vagapova, Evgeniya A. Burilova, Gulnara A. Gaynanova, ${ }^{\circledR}$ \\ Lucia Ya. Zakharova, Alexander R. Burilov, and Michael A. Pudovik \\ Arbuzov Institute of Organic and Physical Chemistry, FRC Kazan Scientific Center of RAS, 420088 Kazan, Russia \\ @Corresponding author E-mail: ggulnara@bk.ru
}

\begin{abstract}
A series of new water-soluble onium salts was obtained by the interaction of aminomethylated calix[4]resorcinol with 1-aminoethylidenediphosphonic acid at reagent ratio of 1:4. To confirm the structure of the compounds, the data of elemental analysis, IR and NMR $\left({ }^{1} \mathrm{H},{ }^{13} \mathrm{C},{ }^{31} \mathrm{P}\right)$ spectroscopy were used. For salt structure with pentyl substituents along the lower rim of the macrocycle the ability to form nanoscale aggregates in an aqueous solution has been shown. Values of the critical association concentration were determined by the methods of tensiometry $\left(9 \cdot 10^{-4} \mathrm{~mol} / \mathrm{L}\right)$ and conductometry $\left(C A C_{1}=9 \cdot 10^{-4} \mathrm{~mol} / \mathrm{L}, C A C_{2}=5 \cdot 10^{-3} \mathrm{~mol} / \mathrm{L}\right)$. Aggregate sizes were determined by means of dynamic light scattering $(4 \mathrm{~nm})$.
\end{abstract}

Keywords: Calix[4]resorcinol, bisphosphonic acid, synthesis, self-aggregation.

\section{Синтез и агрегационные свойства солей на основе аминометилированных каликс[4]резорцинов и 1-аминоэтилидендифосфоновой кислоты}

\author{
^. И. Вагапова, Е. А. Бурилова, Г. А. Гайнанова, ${ }^{@}$ А. Я. Захарова, А. Р. Бурилов, \\ М. А. Пудовик
}

Институт органической и физической химии им. А.Е. Арбузова ФИЦ Казанский научньй центр РАН, 420088 Казань, Россия

${ }^{\circledR}$ E-mail: ggulnara@bk.ru

\begin{abstract}
Серия новых водорастворимых ониевых солей получена взаимодействием аминометилированных каликс[4]резориинов с 1-аминоэтилидендифосфоновой кислотой при соотношении реагентов 1:4. Для подтверждения структуры соединений использовали данные элементного анализа, ИК, ЯМР $\left({ }^{1} \mathrm{H},{ }^{13} \mathrm{C},{ }^{31} \mathrm{P}\right)$ спектроскопии. Для солевой структуры с пентильными заместителями по нижнему ободу макрочикла показана возможность образовывать наноразмерные агрегаты в водном растворе. Определены агрегационные характеристики соли с пентильными заместителями: критическая концентрация ассоциаџии (ККА) по данным методов тензиометрии $\left(9 \cdot 10^{-4} \mathrm{M}\right)$ и кондуктометрии $\left(K K A_{1}=9 \cdot 10^{-4} \mathrm{M}, \mathrm{KK} A_{2}=5 \cdot 10^{-3} \mathrm{M}\right)$, а также размер их ассоичитов по данным метода динамического рассеяния света (4 нм).
\end{abstract}

Ключевые слова: Каликс[4]резорцин, бисфосфоновая кислота, синтез, самоорганизация. 


\section{Introduction}

Supramolecular chemistry has originated from the study of the selective binding of alkali metal cations to macrocyclic ligands, crown ethers and cryptands. ${ }^{[1]}$ Later, the range of objects of supramolecular chemistry was expanded to a wide class of systems connected in a single unit due to intermolecular bonds. ${ }^{[2-5]}$ However, calix[4]resorcinols are widely used as building blocks for supramolecular structures due to the developed synthesis methods, the possibility of functionalization along the upper and lower rims, and also the presence of an internal cavity for guest recognition. ${ }^{[6-9]}$ Modification of calix[4]resorcinol allows to obtain new spatially organized structures with practically useful properties. ${ }^{[10]}$ In addition, the ability to form aggregates (micelles, vesicles) has been shown for amphiphilic calix[4]resorcinol in aqueous, aqueous-organic and organic solvents. ${ }^{[2]}$

Earlier, by Mannich reaction of calix[4] resorcinol with formaldehyde and amines, aminomethylated calix[4]resorcinols were obtained..$^{[1,12]}$ The presence of a hydrophobic cavity in these macrocycles along with four strongly basic dialkylaminomethyl groups allows us to construct new supermolecules that can provide, for example, a catalytic action in reactions of phosphonates hydrolysis,${ }^{[13]}$ and also creates prerequisites for the synthesis of new salt structures with additional centers of coordination. ${ }^{[14]}$

Currently, there is growing interest in obtaining salts of organic molecules based on bisphosphonic acids. ${ }^{[15,16]}$ The ability of these low-toxicity acids to form gels, as well as stable complexes with metals ${ }^{[17-19]}$ allows their using as components of supramolecular gels. ${ }^{[2]}$ In addition, bisphosphonates are recognized as an important class of drugs for the treatment of various diseases associated with excessive bone resorption, including Paget's disease, ${ }^{[2]]}$ bone metastases and osteoporosis. ${ }^{[22]}$ For instance, in ${ }^{[23]}$ it was performed a computer simulation of the correspondence of nine different calix[4] arenes and calix[4]resorcinarenes cavity to the osteoporosis inhibitor zoledronic acid (a third generation of bisphosphonates) and it was found that in the gaseous states some of the complexes may be unstable, but in an aqueous medium almost all complexes can form spontaneously.

In the present work, in order to obtain new watersoluble macrocyclic "hosts" we have studied the interaction of aminomethylated calix[4]resorcinols 1a-c, 2, 3 with 1-aminoethylidenediphosphonic acid 4 (Figure 1). The reaction was carried out at different ratio of reagents (from 1:1 to 1:4). It was found that macrocycles react with acid $\mathbf{4}$ and form tetraonium salts of calix[4] resorcinol 5a-c, 6, 7 regardless of the ratio of the reactants and the order of their mixing.

\section{Experimental}

The general procedure for obtaining compounds $\mathbf{5 a}-\mathbf{c}, \mathbf{6}$, 7 was as follows: to $1.5 \mathrm{mmol}$ of aminomethylated calix[4] resorcinarenes 1a-c, 2 and $\mathbf{3}$ the acid $\mathbf{4}(6 \mathrm{mmol}$ in $200 \mathrm{~mL}$ of water $)$ was added dropwise at constant stirring. The reaction mixture was stirred till complete dissolution of initial reagents and afterwards for $12 \mathrm{~h}$ more, the solvent was distilled off in a vacuum on a rotary evaporator. The residue was washed with acetone and dried in a vacuum of an oil pump. The structure of the synthesized compounds 5a-c, 6 and 7 was confirmed by ${ }^{1} \mathrm{H},{ }^{13} \mathrm{C}$, and ${ }^{31} \mathrm{P}$ NMR spectra, and the composition was confirmed by elemental analysis. NMR spectra were recorded on a Bruker AVANCE 400 spectrometer at $303 \mathrm{~K}$. IR spectra were recorded on a Vector 22 Fourier spectrometer (Bruker, Germany) in the range $400-4000 \mathrm{~cm}^{-1}$. Crystalline samples were studied as suspensions in Nujol. Elemental analyzes were performed on a Carlo Erba elemental analyzer EA 1108.

4,6,10,12,16,18,22,24-Octahydroxy-5,11,17,23-tetrakis[N-diethylaminomethyl]-2,8,14,20-tetramethylpentacyclo[19.3.1.1 $\left.1^{3,7} \cdot 1^{9,13} \cdot 1^{15,19}\right]$ octacosa-1(25),3,5,7(28),9,11,13(27), 15,17, 19(26),21,23-dodecaene- $N, N, N, N$-tetrakis (hydro(1-amino1-phosphonoethyl)phosphonate) (5a). Slightly pink powder, yield $92 \%$. M.p. $234-236{ }^{\circ} \mathrm{C}$ decomp. Anal. Calcd for $\mathrm{C}_{60} \mathrm{H}_{112} \mathrm{~N}_{8} \mathrm{O}_{32} \mathrm{P}_{8}$ : C 42.25, H 6.57, N 6.57, P 14.55. Found: C 42.10, H 6.62, N 6.59, $\mathrm{P}$ 14.50. IR (nujol) $\mathrm{v}_{\max } \mathrm{cm}^{-1}: \mathrm{m} 1056(\mathrm{POH}), \mathrm{s} 1166,1232(\mathrm{P}=\mathrm{O})$, $1605(\mathrm{C}=\mathrm{Car})$, br $2268-2538\left(\mathrm{NH}^{+}\right)$, br $3135,3382\left(\mathrm{OH}, \mathrm{NH}_{2}\right) \cdot{ }^{31} \mathrm{P}$ NMR (D O) $\delta_{\mathrm{p}}$ ppm: 13.2. ${ }^{1} \mathrm{H}$ NMR $\left(600 \mathrm{MHz}, \mathrm{D}_{2} \mathrm{O}\right) \delta_{\mathrm{H}} \mathrm{ppm}: 1.25$ $\left(\mathrm{t}, 24 \mathrm{H}, J=7.2 \mathrm{~Hz}, \mathrm{CH}_{3}\right), 1.39\left(\mathrm{~d}, 12 \mathrm{H}, J=6.9 \mathrm{~Hz}, \mathrm{CHCH}_{3}\right), 1.57$ (d, $\left.6 \mathrm{H},{ }^{3} J_{\mathrm{PH}}=13.9 \mathrm{~Hz}, \mathrm{PCCH}_{3}\right), 1.59\left(\mathrm{~d}, 6 \mathrm{H},{ }^{3} \mathrm{P}_{\mathrm{PH}}=14.0 \mathrm{~Hz}, \mathrm{PCCH}_{3}\right)$,

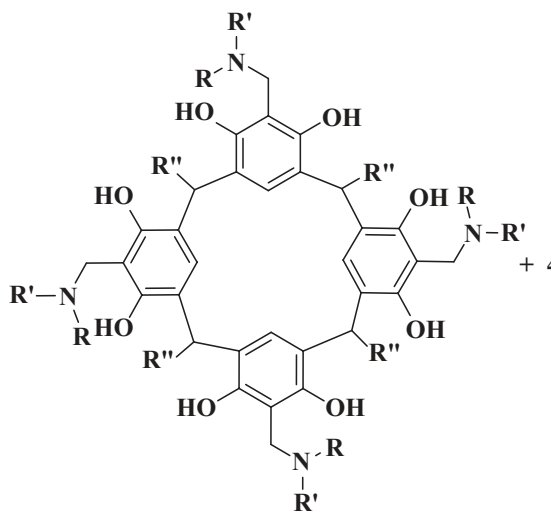

1a-c, 2, 3

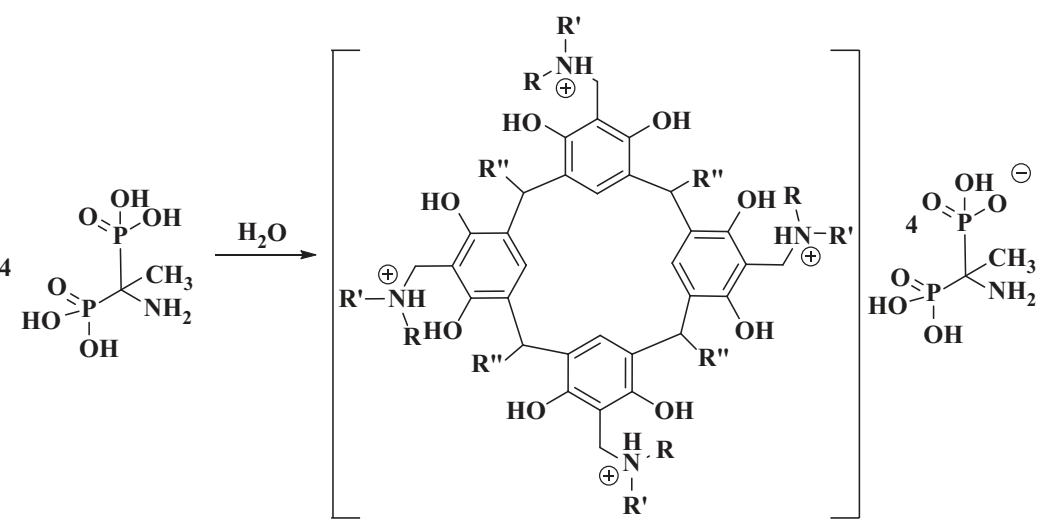

4

5a-c, 6, 7

1a-c, 5a-c (R=R'=Et, $\mathrm{R}^{\prime \prime}=\mathrm{Me}(\mathrm{a}), \operatorname{Et}(\mathrm{b}), \mathrm{C}_{5} \mathrm{H}_{11}(\mathrm{c})$;

2, $6\left(\mathrm{R}=\mathrm{R}^{\prime}=\mathrm{Me}, \mathrm{R}^{\prime \prime}=\mathrm{Me}\right)$;

3, $7\left(\mathrm{R}=\mathrm{Me}, \mathrm{R}^{\prime}=\mathrm{CH}_{2} \mathrm{CH}(\mathrm{OMe})_{2}, \mathrm{R}^{\prime \prime}=\mathrm{Me}\right)$

Figure 1. The interaction of aminomethylated calix[4]resorcinols with 1-aminoethylidenediphosphonic acid. 
3.09-3.14 (m, 16H, $\left.\mathrm{NCH}_{2} \mathrm{CH}_{3}\right), 4.22\left(\mathrm{~s}, 8 \mathrm{H}, \mathrm{NCH}_{2}\right), 4.39-4.44$ (q, $4 \mathrm{H}, J=6.9 \mathrm{~Hz}, \mathrm{Ar}-\mathrm{CH}), 6.71$ (s, $4 \mathrm{H}, \mathrm{Ar}-\mathrm{H}) .{ }^{13} \mathrm{C} \mathrm{NMR}(100.6 \mathrm{MHz}$, $\left.\mathrm{D}_{2} \mathrm{O}\right) \delta_{\mathrm{C}}$ ppm: $8.21\left(\mathrm{CH}_{3}\right), 17.69\left(\mathrm{CH}_{3}\right), 19.88\left(\mathrm{CH}_{3}\right), 31.28(\mathrm{CH})$, $46.15\left(\mathrm{CH}_{2} \mathrm{Ar}\right), 47.71\left(\mathrm{CH}_{2} \mathrm{~N}\right), 53.99 \mathrm{t}\left(\mathrm{P}-\mathrm{C}-\mathrm{CH}_{3}\right), 109.44\left(\mathrm{C}_{\mathrm{ar}}-\mathrm{CH}\right)$, 126. $53(\mathrm{C}-\mathrm{CH}), 126.67\left(\mathrm{C}_{\mathrm{ar}} \mathrm{H}\right), 150.99(\mathrm{C}-\mathrm{OH})$.

4,6,10,12,16,18,22,24-Octahydroxy-5,11,17,23-tetrakis[N-diethylaminomethyl]-2, 8,14,20-tetraethylpentacy clo[19.3.1.1 $\left.1^{3,7} \cdot 1^{9,13} \cdot 1^{15,19}\right]$ octacosa-1(25),3,5,7(28),9,11,13(27),15, 17,19(26),21,23-dodecaene- $N, N, N, N$-tetrakis(hydro(1-amino-1-phosphonoethyl)phosphonate) (5b). Slightly pink powder, yield $92 \%$. M.p. $>250{ }^{\circ} \mathrm{C}$ decomp. Anal. Calcd for $\mathrm{C}_{64} \mathrm{H}_{120} \mathrm{~N}_{8} \mathrm{O}_{32} \mathrm{P}_{8}$ $\left(\mathrm{C}_{56} \mathrm{H}_{84} \mathrm{~N}_{4} \mathrm{O}_{8} \times 4 \mathrm{C}_{2} \mathrm{H}_{9} \mathrm{NO}_{6} \mathrm{P}_{2}\right): \mathrm{C} 43.62, \mathrm{H} 6.82, \mathrm{~N} 6.36, \mathrm{P} 14.09$. Found: C 43.34, H 6.68, N 6.37, P 14.17. IR (nujol) $v_{\max } \mathrm{cm}^{-1}: \mathrm{m}$ $1054(\mathrm{POH}), \mathrm{s} 1165,1232(\mathrm{P}=\mathrm{O}), 1605(\mathrm{C}=\mathrm{Car})$, br $2268-2530$ $\left(\mathrm{NH}^{+}\right)$, br 3240, $3415(\mathrm{NH}, \mathrm{OH}) .{ }^{31} \mathrm{P}$ NMR $\left(\mathrm{D}_{2} \mathrm{O}\right) \delta_{\mathrm{p}} \mathrm{ppm}: 12.9 .{ }^{1} \mathrm{H}$ NMR $\left(600 \mathrm{MHz}, \mathrm{D}_{2} \mathrm{O}\right) \delta_{\mathrm{H}}$ ppm: $0.86\left(\mathrm{t}, 12 \mathrm{H}, J=7.2 \mathrm{~Hz}, \mathrm{CH}_{3}\right), 1.18$ $\left(\mathrm{m}, 24 \mathrm{H}, J=7.2 \mathrm{~Hz}, \mathrm{CH}_{2}\right), 1.58\left(\mathrm{~d}, 6 \mathrm{H},{ }^{3} J_{\mathrm{PH}}=13.9 \mathrm{~Hz}, \mathrm{PCCH}_{3}\right)$, $1.61\left(\mathrm{~d}, 6 \mathrm{H},{ }^{3} \mathrm{JH}_{\mathrm{PH}}=13.9 \mathrm{~Hz}, \mathrm{PCCH}_{3}\right), 2.12-2.18\left(\mathrm{~m}, 8 \mathrm{H}, \mathrm{CHCH}_{2}\right)$, 3.04-3.06 (m, $\left.16 \mathrm{H}, \mathrm{NCH}_{2} \mathrm{CH}_{3}\right), 4.22\left(\mathrm{~m}, 8 \mathrm{H}, \mathrm{NCH}_{2}, 4 \mathrm{H}, \mathrm{Ar}-\mathrm{CH}\right)$, 7.28 (s, 4H, Ar-H).

4, 6,10,12,16,18,22,24-Octahydroxy-5,11,17,23-tetrakis[N-diethylaminomethyl]-2,8,14,20-tetrapentylpentacyclo[19.3.1.1 $\left.1^{3,7} \cdot 1^{9,13} \cdot 1^{15,19}\right]$ octacosa-1(25), 3, 5,7(28),9,11,13(27), 15,17, 19(26),21,23-dodecaene- $N, N, N, N$-tetrakis (hydro(1-amino-1-phosphonoethyl)phosphonate) (5c). Slightly pink powder, yield $96 \%$. M.p. $>350{ }^{\circ} \mathrm{C}$. Anal. Calcd for $\mathrm{C}_{76} \mathrm{H}_{144} \mathrm{~N}_{8} \mathrm{O}_{32} \mathrm{P}_{8}$ $\left(\mathrm{C}_{68} \mathrm{H}_{108} \mathrm{~N}_{4} \mathrm{O}_{8} \times 4 \mathrm{C}_{2} \mathrm{H}_{9} \mathrm{NO}_{6} \mathrm{P}_{2}\right): \mathrm{C} 47.30, \mathrm{H} 7.47, \mathrm{~N}$ 5.81, P 12.86 . Found: C 47.14, H 7.48, N 5.77, P 12.94. IR (nujol) $v_{\max } \mathrm{cm}^{-1}: \mathrm{m} 1052$ $(\mathrm{POH}), \mathrm{s} 1165,1232(\mathrm{P}=\mathrm{O}), 1603(\mathrm{C}=\mathrm{Car})$, br $2368-2530\left(\mathrm{NH}^{+}\right)$, br $3230,3420(\mathrm{OH}) .{ }^{31} \mathrm{P}$ NMR $\left(\mathrm{D}_{2} \mathrm{O}\right) \delta_{\mathrm{P}} \mathrm{ppm}: 13.40 .{ }^{1} \mathrm{H}$ NMR (600 MHz, $\left.\mathrm{D}_{2} \mathrm{O}\right) \delta_{\mathrm{H}}$ ppm: 0.78 (br.s, $\left.12 \mathrm{H}, \mathrm{CH}_{3}\right), 1.14-1.20(\mathrm{~m}, 40 \mathrm{H}$, $\left.\left.\mathrm{N}\left(\mathrm{CH}_{2} \mathrm{CH}_{3}\right)_{2},\left(\mathrm{CH}_{2}\right)_{3}\right), 1.59\left(\mathrm{~d}, 6 \mathrm{H},{ }^{3} J_{\mathrm{PH}}=13.7 \mathrm{~Hz}, \mathrm{PCCH}\right)_{3}\right), 1.60(\mathrm{~d}$, $6 \mathrm{H},{ }^{3} \mathrm{~J}_{\mathrm{PH}}=14.0 \mathrm{~Hz}, \mathrm{PCCH}$ ), 1.91 (br.s, $8 \mathrm{H}, \mathrm{CHCH}_{2}$ ), 3.01-3.05 (m, $16 \mathrm{H}, \mathrm{NCH}_{2} \mathrm{CH}_{3}$ ), 4.23 (br.s, 8H, $\mathrm{NCH}_{2}$ ), 4.36 (br.s, $4 \mathrm{H}, \mathrm{Ar}-\mathrm{CH}$ ), 5.24 (s, 2H, Ar-H), 6.65 (s, 2H, Ar-H).

4,6,10,12,16,18,22,24-Octahydroxy-5,11,17,23-tetrakis[N-dimethylaminomethyl]-2,8,14,20-tetramethylpentacyclo$\left[19.3 \cdot 1 \cdot 1^{3,7} \cdot 1^{9,13} \cdot 1^{15,19}\right]$ octacosa-1(25), 3, 5, 7(28),9,11,13(27), 15,17, 19(26),21,23-dodecaene- $N, N, N, N$-tetrakis (hydro(1-amino-1-phosphonoethyl)phosphonate) (6). Slightly pink powder, yield $92 \%$. M.p. $>250{ }^{\circ} \mathrm{C}$ decomp. Anal. Calcd for $\mathrm{C}_{52} \mathrm{H}_{60} \mathrm{~N}_{4} \mathrm{O}_{8}$ $\left(\mathrm{C}_{44} \mathrm{H}_{60} \mathrm{~N}_{4} \mathrm{O}_{8} \times 4 \mathrm{C}_{2} \mathrm{H}_{9} \mathrm{NO}_{6} \mathrm{P}_{2}\right): \mathrm{C} 39.20, \mathrm{H} 6.03, \mathrm{~N}$ 7.03, $\mathrm{P} 15.55$. Found: C 38.99, H 6.10, N 5.67, P 15.55. IR (nujol) $v_{\max } \mathrm{cm}^{-1}: \mathrm{m}$ $1054(\mathrm{POH}), \mathrm{s} 1156,1232(\mathrm{P}=\mathrm{O}), 1598(\mathrm{C}=\mathrm{Car})$, br $2268-2530$ $\left(\mathrm{NH}^{+}\right)$, br 3135, $3384(\mathrm{OH}) .{ }^{31} \mathrm{P}$ NMR $\left(\mathrm{D}_{2} \mathrm{O}\right) \delta_{\mathrm{p}} \mathrm{ppm}:$ 13.7. ${ }^{1} \mathrm{H}$ NMR $\left(600 \mathrm{MHz}, \mathrm{D}_{2} \mathrm{O}\right) \delta_{\mathrm{H}} \mathrm{ppm}: 1.43\left(\mathrm{~d}, 12 \mathrm{H}, J=6.7 \mathrm{~Hz}, \mathrm{CH}_{3}\right), 1.58(\mathrm{~d}$, $\left.6 \mathrm{H},{ }^{3} \mathrm{~J}_{\mathrm{PH}}=13.8 \mathrm{~Hz}, \mathrm{PCCH}_{3}\right), 1.60\left(\mathrm{~d}, 6 \mathrm{H},{ }^{3} \mathrm{~J}_{\mathrm{PH}}=13.8 \mathrm{~Hz}, \mathrm{PCCH}_{3}\right)$, $2.78\left(\mathrm{~s}, 24 \mathrm{H}, \mathrm{NCH}_{3}\right), 4.26\left(\mathrm{~s}, 8 \mathrm{H}, \mathrm{NCH}_{2}\right), 4.45$ (q, $4 \mathrm{H}, J=6.7 \mathrm{~Hz}$, Ar- $\mathrm{CH}), 6.76$ (s, $4 \mathrm{H}, \mathrm{Ar}-\mathrm{H})$.

4, 6,10,12,16,18,22,24-Octahydroxy-5,11,17,23-tetrakis[(N-(2,2-dimethoxyethyl)(methyl)amino)methyl $]-2,8,14,20$ tetramethylpentacyclo[19.3.1.1 $\left.1^{3,7} \cdot 1^{9,13} \cdot 1^{15,19}\right]$ octacosa-1(25),3, 5,7(28),9,11,13(27),15,17,19(26),21,23-dodecaene- $N, N, N, N$ -tetrakis(hydro(1-amino-1-phosphonoethyl)phosphonate) (7). Slightly pink powder, yield $96 \%$. M.p. $168-179{ }^{\circ} \mathrm{C}$ decomp. Anal. Calcd for $\mathrm{C}_{64} \mathrm{H}_{120} \mathrm{~N}_{8} \mathrm{O}_{40} \mathrm{P}_{8}\left(\mathrm{C}_{56} \mathrm{H}_{84} \mathrm{~N}_{4} \mathrm{O}_{16} \times 4 \mathrm{C}_{2} \mathrm{H}_{9} \mathrm{NO}_{6} \mathrm{P}_{2}\right)$ : C $40.68, \mathrm{H}$ 6.40, N 5.93, P 13.11. Found: C 40.58, H 6.34, N 5.82, P 13.22. IR (nujol) $v_{\max } \mathrm{cm}^{-1}: \mathrm{m} 1054(\mathrm{POH}), \mathrm{s} 1156,1232(\mathrm{P}=\mathrm{O}), 1598$ $(\mathrm{C}=\mathrm{Car})$, br $2268-2530\left(\mathrm{NH}^{+}\right)$, br 3135, $3384(\mathrm{OH}) .{ }^{31} \mathrm{P} \mathrm{NMR}\left(\mathrm{D}_{2} \mathrm{O}\right)$ $\delta_{\mathrm{P}}$ ppm: 12.9. ${ }^{1} \mathrm{H}$ NMR $\left(400 \mathrm{MHz}, \mathrm{D}_{2} \mathrm{O}\right) \delta_{\mathrm{H}} \mathrm{ppm}: 1.49(\mathrm{~d}, 12 \mathrm{H}$, $\left.J=6.7 \mathrm{~Hz}, \mathrm{CH}_{3}\right), 1.56\left(\mathrm{~d}, 6 \mathrm{H},{ }^{3} J_{\mathrm{PH}}=13.8 \mathrm{~Hz}, \mathrm{PCCH}_{3}\right), 1.59(\mathrm{~d}$, $\left.6 \mathrm{H},{ }^{3} J_{\mathrm{PH}}=13.8 \mathrm{~Hz}, \mathrm{PCCH}\right), 2.81\left(\mathrm{~s}, 12 \mathrm{H}, \mathrm{NCH}_{3}\right), 3.22$ (br.s $32 \mathrm{H}$, $\mathrm{OCH}_{3}, \mathrm{NCH}_{2} \mathrm{CH}$ ), 4.36 (br. s, $8 \mathrm{H}, \mathrm{NCH}_{2}$ ), 4.45 (q, $4 \mathrm{H}, J=6.7 \mathrm{~Hz}$, $\mathrm{Ar}-\mathrm{CH}), 4.59$ (m, 4H, CHOMe), 6.96 (s, 4H, Ar-H). ${ }^{13} \mathrm{C}$ NMR $\left(100.6 \mathrm{MHz}, \mathrm{D}_{2} \mathrm{O}\right) \delta_{\mathrm{C}}, \mathrm{ppm}: 17.65\left(\mathrm{CH}_{3}\right), 19.51(\mathrm{CH}), 30.62\left(\mathrm{NCH}_{3}\right)$, $42.35\left(\mathrm{CH}_{2} \mathrm{Ar}\right), 51.39\left(\mathrm{CH}_{2} \mathrm{~N}\right), 53.96\left(\mathrm{P}-\mathrm{C}-\mathrm{CH}_{3}\right), 55.48\left(\mathrm{NCH}_{2}\right)$, $56.43\left(\mathrm{OCH}_{3}\right), 99.64\left(\mathrm{C}_{\mathrm{ar}}{ }^{2} \mathrm{CH}\right), 108.29(\mathrm{C}-\mathrm{CH}), 126.94\left(\mathrm{C}_{\mathrm{ar}} \mathrm{H}\right)$, $150.95(\mathrm{C}-\mathrm{OH})$.
Purified water $\left(18.2 \mathrm{M} \Omega \mathrm{cm}\right.$ resistivity at $\left.25^{\circ} \mathrm{C}\right)$ from DirectQ 5 UV equipment was used for all solution preparation.

Surface tension measurements were performed using the du Nouy ring detachment method with the tensiometer K6 (Kruss, Germany). The measurements were carried out in a thermostatic cell manually until a stable value was obtained on the surface of $10 \mathrm{~mL}$ solutions. The concentration dependence of the surface tension represents the average value of at least five measurements, and the error was within $2 \%$

The conductivity $(\chi)$ was measured on the InoLab Cond 7110 conductometer (WTW, Germany) with a TetraCon remote sensor.

Aggregates size was measured by means of dynamic light scattering with ZetaSizer Nano system (Malvern, UK).

\section{Results and Discussion}

The obtained compounds are of interest as promising preorganized structures for the design of organized systems with controlled properties, ligands, supramolecular catalysts. The presence in the structure of the synthesized macrocycles $\mathbf{1 a}-\mathbf{c}, \mathbf{2}$ and $\mathbf{3}$ of hydrophilic groups on the upper rim along with a hydrophobic cavity and alkyl substituents suggests their amphiphilic character. It is known that the aggregation of calix[4]resorcinol depends on the length of the alkyl substituents on the lower rim of the macrocycles. ${ }^{[2]}$ Therefore, for the onium salts $\mathbf{5 a}$ and $\mathbf{5 c}$, the possibility of the aggregates formation in an aqueous solution was studied by the methods of tensiometry and conductometry, and the values of the critical association concentration (CAC) were determined from the break points at the corresponding concentration dependences. Both complexes 5a and $\mathbf{5 c}$ have good solubility in water.

On the surface tension isotherm of compound $\mathbf{5 a}$ (Figure 2a), a slight decrease in the surface tension up to $58 \mathrm{mN} / \mathrm{m}$ is observed without a pronounced exit to the plateau, which does not allow us to identify the value of CAC. This can be explained by a short (methyl) tail on the lower rim of aminomethylated calix[4]resorcinol. The concentration dependence of the specific conductivity (Figure 3a) shows a smooth increase associated with an increase in the number of charge carriers with break point at concentration of 5a equal to $0.01 \mathrm{~mol} / \mathrm{L}$.

The presence of a pentyl substituent on the lower rim of aminomethylated calixarene allowed us to suggest the possibility of the formation of aggregates in $\mathbf{5 c}$ solution. From the surface tension isotherm (Figure 2b) it can be seen that, compared to compound $\mathbf{5 a}$ for salt with pentyl substituents $\mathbf{5} \mathbf{c}$ the dependence goes to a plateau. The value of CAC for compound $\mathbf{5 c}$ is $9 \cdot 10^{-4} \mathrm{~mol} / \mathrm{L}$. However, it is worth noting that for classical surfactants in aqueous solutions, it is possible to achieve a much larger decrease in surface tension up to $30-35 \mathrm{mN} / \mathrm{m}$. The location of the plateau region for compound $\mathbf{5 c}$ at values of $45-47 \mathrm{mN} / \mathrm{m}$ is most likely due to the bulk structure of the onium salt molecule, which prevents its large accumulation in the surface layer.

On the dependence of the specific conductivity (Figure $3 b$ ) on the concentration of compound $\mathbf{5 c}$, there are two inflections: $\mathrm{CAC}_{1}\left(9 \cdot 10^{-4} \mathrm{~mol} / \mathrm{L}\right)$ can be attributed to the beginning of the formation of aggregates in solution, and $\mathrm{CAC}_{2}\left(5 \cdot 10^{-3} \mathrm{~mol} / \mathrm{L}\right)$ can be attributed to structural rearrangements in the system. The hydrodynamic diameter of the aggregates, determined by dynamic light scattering, 

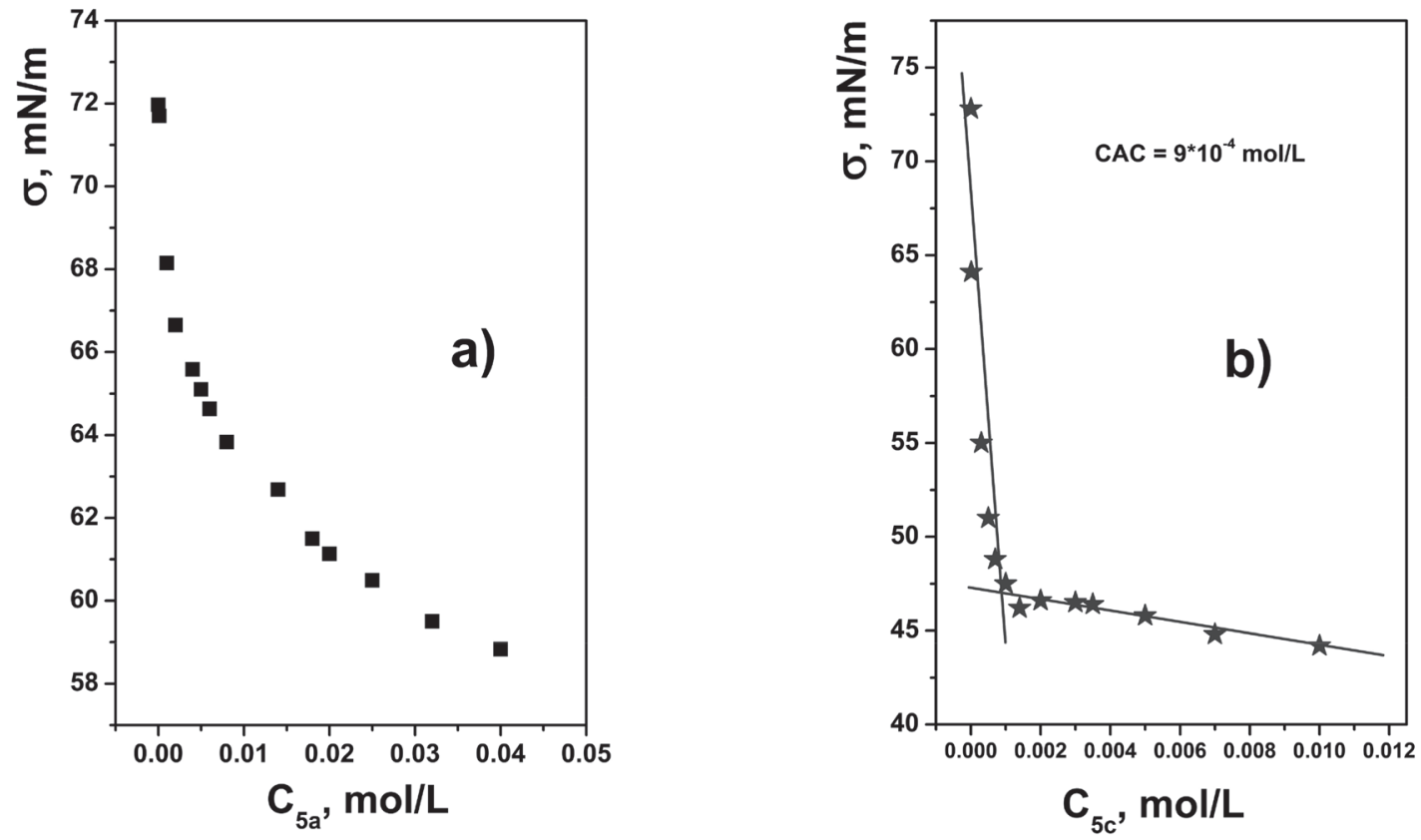

Figure 2. Surface tension isotherms of aqueous solutions of compounds $\mathbf{5 a}$ (a) and $\mathbf{5 c}(\mathrm{b}), 25^{\circ} \mathrm{C}$.
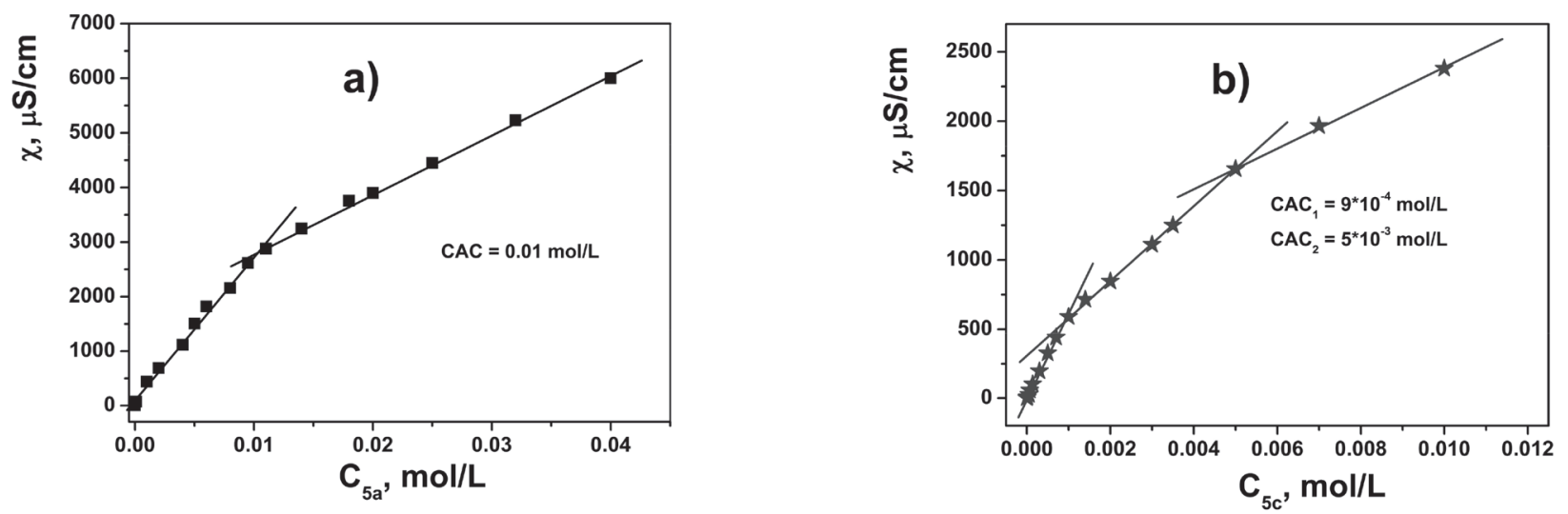

Figure 3. The specific conductivity versus concentration of onium salts $\mathbf{5 a}$ (a) and $\mathbf{5 c}$ (b), aqueous solution, $25^{\circ} \mathrm{C}$.

in an aqueous solution of $\mathbf{5 c}$ was $4 \mathrm{~nm}$ in the entire concentration range higher than the values of $\mathrm{CAC}_{1}$. Constant diameter value indicates that, as a result of the reconstruction of the aggregates after $\mathrm{CAC}_{2}$, the aggregates do not enlarge, but, most likely, their packing is becoming denser.

\section{Conclusions}

The first representatives of new water-soluble onium salts based on aminomethylated calix[4]resorcinol having different hydrophobicity and 1-aminoethylidenediphosphonic acid were obtained by varying the ratio of reagents (from 1:1 to 1:4). The structure of the compounds formed was confirmed by elemental analysis, IR, NMR $\left({ }^{1} \mathrm{H},{ }^{13} \mathrm{C}\right.$, $\left.{ }^{31} \mathrm{P}\right)$ spectroscopy. For a salt structure with pentyl substituents along the lower rim of the macrocycle, the possibility of the formation of nanoscale aggregates in an aqueous solution was shown. Values of the critical association concentration were determined by tensiometry $\left(9 \cdot 10^{-4} \mathrm{~mol} / \mathrm{L}\right)$ and conductometry $\left(\mathrm{CAC}_{1}=9 \cdot 10^{-4} \mathrm{~mol} / \mathrm{L}, \mathrm{CAC}_{2}=5 \cdot 10^{-3} \mathrm{~mol} / \mathrm{L}\right)$.

\section{References}

1. Lehn J.-M. Supramolecular Chemistry. Concepts and Perspectives (russ. transl.). Novosibirsk: Nauka. Sib. predpriyatiye RAN, 1998. 334 p.

2. Razuvayeva Yu., Kashapov R., Zakharova L. Supramol. Chem. 2020, 32, 178-206.

3. Basilio N., Gomez B., Garcia-Rio L., Francisco V. Chem. Eur. J. 2013, 19, 4570-4576.

4. Kashapov R.R., Razuvayeva Y.S., Ziganshina A.Y., Mukhitova R.K., Sapunova A.S., Voloshina A.D., Zakharova L.Ya. Macroheterocycles 2019, 12, 346-349. 
5. Costa C., Francisco V., Silva S.G., do Vale M.L.C., GarciaRio L., Marques E.F. Colloids Surf., A: Physicochem. Eng. Aspects 2015, 480, 71-78.

6. Timmerman P., Verboom W., Reinhoudt D.N. Tetrahedron 1996, 52, 2663-2704.

7. Jain V.K., Kanaiya P.H. Russ. Chem. Rev. 2011, 80, 75102.

8. Burilov V.A., Garipova R.I., Sultanova E.D., Mironova D.A., Evtugyn V.G., Osin Yu.N., Solovieva S.E., Antipin I.S. Macroheterocycles 2019, 12, 340-345.

9. Sansone F., Baldini L., Casnati A., Ungaro R. New J. Chem. 2010, 34, 2715-2728.

10. Li N., Harrison R.G., Lamb J.D. J. Inclusion Phenom. Macrocycl. Chem. 2014, 78, 39-60.

11. Vagapova L.I., Fahertdinova A.F., Burilov A.R., Pudovik M.A. Mendeleev Commun. 2012, 22, 325-326.

12. Vagapova L.I., Burilov A.R., Voronina Yu.K., Syakaev V.V., Sharafutdinova D.R., Amirova L.R., Pudovik M.A., Garifzyanov A.R., Sinyashin O.G. Heteroat. Chem. 2014, 25, 178-185.

13. Pashirova T.N., Lukashenko S.S., Kosacheva E.M., Leonova M.V., Vagapova L.I., Burilov A.R., Pudovik M.A.,
Kudryavtseva L.A., Konovalov A.I. Russ. J. Gen. Chem. 2008, 78, 402-409.

14. Vagapova L.I., Nasirova Z.A., Burilova E.A., Zobov V.V., Burilov A.R., Amirov R.R., Pudovik M.A. Russ. J. Org. Chem. 2017, 53, 312-314.

15. Chen S.-P., Hu L., Zhang Y.-Q., Deng P., Li C., Chen X., Yuan L.-J. Chem. Commun. 2012, 48, 552-554.

16. Chen S.-P., Yuan Y.-X., Pan L.-L., Xu S.-Q., Xia H., Yuan L.-J. Cryst. Growth 2009, 9, 874-879.

17. Rizkalla E.N., Zaki M.T.M., Ismail M.I. Talanta 1980, 27, 715-719.

18. Cilley W.A., Grabenstetter R.J. J. Phys. Chem. 1971, 75, 676-682.

19. Ding D.-G., Zhang X. Synth. React. Inorg. Met.-Org. NanoMet. Chem. 2012, 8, 1087-1090.

20. Xu K., Ge W., Liang G., Wang L., Yang Z., Wang Q., Hsing I.-M., Xu B. Int. J. Radiat. Biol. 2008, 84, 353-362.

21. Cundy T. Metab.: Clin. Exp. 2018, 80, 5-14.

22. Gennari L., Bilezikian J.P. J. Exp. Opin. Pharmacoth. 2018, $19,253-264$

23. Jang Y.-M., Yu Ch.-J., Kim J.-S., Kim S.-U. J. Mater. Sci. 2018, 53, 5125-5139. 